(From the Physiological Laboratory of the University of California, Berkeley, California.)

\title{
Ueber den segmentalen Charakter des Athemcentrums in der Medulla oblongata der Warmblüter.
}

Von

Jacques Loeb.

1. Die schönen Versuche von Grünbaum und Sherrington haben eine regelmässige Anordnung der Reizstellen in der Grosshirnrinde des Affen ergeben, welche der Anordnung der Rückenmarkssegmente in hohem Grade entspricht ${ }^{1}$ ). Diese Thatsache unterstützt sehr wesentlich die segmentale Theorie der Functionen des Nervensystems, d. h. die Vorstellung, dass bei höheren Thieren, wie bei den niederen, das Centralnervensystem im Wesentlichen nur eine Reihe von segmentalen Ganglien bildet. Man weiss, dass dieser auf die vergleichende Physiologie sich stützenden Anschauung eine andere Auffassung, nämlich die Centrentheorie, gegenübersteht, wonach complicirte Functionen des gesammten Körpers in einem beschränkten Bezirk des Gehirns oder der Medulla oblongata localisirt sind. Ich habe die Gründe, welche für die segmentale Theorie auch bei höheren Thieren sprechen, in der deutschen und noch eingehender in der englischeu Ausgabe meiner vergleichenden Gehirnphysiologie auseinandergesetzt. Da nun neuerdings die Arbeiten von $\mathrm{Babák}^{2}$ ) und Philippson ${ }^{3}$ ) zeigen, dass das Interesse für die segmentale Auffassung des Centralnervensystems im Zunehmen begriffen ist, so will ich in Folgendem kurz darauf hinweisen, dass auch eine bekannte, scheinbar widersprechende Thatsache

1) Grünbaum und Sherrington, Proceedings of the Royal Society rol. 69 p. 206.1901.

2) Babák, Dieses Archiv Bd. 93 S. 134.1902.

3) Philippson, Comptes rendus de l'académie des sciences vol. 136 p. 61 . 1903 . 
sich dieser Theorie unterordnen lässt, nämlich die Existenz eines Athemcentrums in der Medulla oblongata der Warmblüter. Die eigentlichen $\Lambda$ themmuskeln sind bekanntlich die Rippenmuskeln und das Zwerchfell, und deren segmentale Ganglien liegen alle im Hals- und Brustmark. Nichslestoweniger hören alle Athembewegungen auf, wenn man einen Schnitt durch das Rückenmark zwischen Medulla oblongata und Phrenicusursprung macht. Zwei Dinge bleiben hierbei vom Standpunkt der Segmentaltheorie unerklärt: erstens, wie es kommt, dass in der Medulla oblongata ein "Atbemcentrum" existirt, ohne dass demselben ein zu denselben Segmenten gehöriges peripheres Athemorgan entspricht, und zweitens, wie es kommt, dass nach Zerstörung dieses "Athemcentrums" resp. nach Abtrennung des Rückenmarkes von demselhen die automatische Thätigkeit der unterhalb des Schnittes gelegenen segmentalen Athemganglien bei Warmblütern aufhört. Die Centrentheorie baut eben hierauf ibre Idee, dass alle "Functionen" im Gehirn oder der Medulla oblongata centralisirt sind. Dass bei niederen Thieren die Thatsachen dieser Auffassung direct widersprechen, wird mit der Annahme beschwichtigt, dass bei niederen Thieren die Segmentaltheorie richtig sein möge, dass aber bei höheren Thieren, „die "Functionen höber hinaufwandern". Man versuche aber einmal sich eine sinnliche Vorstellung von einer "Wanderung der Functionen" im Centralnervensystem zu bilden, und man wird finden, dass diese Ausdrucksform nur unsere Unkenntniss der thatsächlichen Verhältnisse verschleiern, aber nicht beseitigen kann.

2. Ich habe in meiner "Vergleichenden Gehirnphysiologie" darauf hingewiesen, dass wir auf die embryonalen Zustände bei höheren Thieren zurückgehen müssen, wenn wir zum richtigen Verständniss der Beziehungen des Centralnervensystems zu den peripheren Organen kommen wollen. Der Grund hierfür liegt in der Thatsache, dass die peripheren Organe während der embryonalen Entwicklung viel eingehendere Verschiebungen und Umwandlungen erfahren als die entsprechenden Rückenmarkstheile. Ziehen wir aber die Umwandlungen und die Verschiebungen der peripheren Organe während der embryonalen Entwicklung in Betracht, so finden wir, dass auch bei höheren (wie bei niederen) Thieren die segmentale Anordnung der Innervationsverhältnisse gewahrt bleibt. Als ein schlagendes Beispiel hierfür führte ich die Beziehung des Phrenicus zum Zwerchfell an. Der Phrenicus entspringt im Halsmark, und ehe man die 
embryologische Anlage und Verschiebung des Zwerchfells kannte, konnte man sich vorstellen, dass auch hier ein Widerspruch mit der Segmentaltheorie vorliege, indem das den Boden des Thorax bildende Zwerchfell von einem hoch oben in Halsmark gelegenen "Centrun" innervirt werde. Die embryologische Beobachtung aber hat ergeben, dass das Zwerchfell in denjenigen Segnenten des embryonalen Körpers gebildet wird, in dem der Phrenicus entspringt, und dass nur secundäre embryonale Wachsthumsvorgänge das Zwerchfell aus seinem Entstehungsort nach dem Boden des Thorax drängen. Nur die Nichtberiucksichtigung oder Unkenntniss der embryologischen Thatsachen konnte also hier eine Abweichung von der Segmentaltheorie vermuthen.

Genau so liegen aber die Ininge meines Erachtens in Bezug auf das Athemcentrum in der Medulla oblongata. Beim Erwachsenen entspricht allerdings diesem "Centrum" kein peripheres, segmentales Athemorgan; wenn wir uns dagegen an den Fubryo wenden, so finden wir, wie ich glaube, ein solches. Ich halte es für möglich, dass das A themcentrum in der Medulla oblongata das segmentale Ganglion-oder die segmentalen Ganglien - für ein peripheres Athemorgan enthäl, welches nur im embryonalen Leben des Menschen vorübergehond existirt, nämlich die Kiemen.

Wenn das richtig ist, so ist zu erwarten, dass die Nerven für die Kiemen - oder richtiger Athemmuskeln - bei Fischen der Vagusgruppe angehören, was auch thatsächlich der Fall ist. Ausserdem folgt aber aus der Arbeit von C. J. Herrick'), dass die Athemganglien in der Medulla oblonqata bei Warmblütern völlig homolog, wenn nicht identisch sind mit den segmentalen Ganglien für die Innervation der Kiemen bei Fischen im lobus vagi ${ }^{2}$ ). Der wesentliche Unterschied zwischen den Athemganglien in der Medulla oblongata bei Fischen und Warmblütern ist das Fehlen der Faserzüge zu den Phrenicuskernen bei Fischen, und damit auch das Fehlen des sogen. noeud vital. Da bei Fischen eben die Athmung auf die Kiemen beschränkt ist und keine Athemorgane in den tiefer gelegenen Segmenten existiren, so ist natürlich kein sogen. noeud vital bei denselben mög-

1) C. J. Herrick, The Journal of Comparative Neurology vol. 9 p. 153. 1899.

2) Herr Dr. Hardesty war so treundlich, auf meine Veranlassung den letzteren Punkt zu verificiren. 
lich $\left.{ }^{1}\right)$. Die mit Reclit viel bewunderte Entdeckung embryonaler Kiemen durch Rathke liefert also auch die Stütze für die segmentale Auffassung des Athemcentrums in der Medulla oblongata der Warmblüter.

3. Das "Athemcentrum" in der Medulla oblongata ist also, wenn wir die embryologischen Thatsachen berücksichtigen - und das ist in der Gehirnphysiologie unerlässlich - kcin „übergeordnetes Centrun!" sondern ein segmentales Ganglion, oder vielmehr eine Reihe von segmentalen Ganglien. Dann aber bleilbt noch eine Schwierigkeit bestehen, nämlich wie es kommt, dass die Phrenicuskerne und die Ganglien der costalen Athemmuskeln nicht mehr antomatisch thätig sind, wenn sie von den Athemganglien (den Kiemenganglien) der Medulla oblongata getrennt sind oder wenn die letzteren zersört sind.

Ich bin geneigt anzunehmen, dass auf diesem Gebiet noch theoretisch und für die Praxis wichtige Entdeckungen zu machen sind, bei denen sich möglicher Weise herausstellen wird, dass die Dinge für die verschiedenen Athemganglien ähnlich liegen, wie für die verschiedenen Abschnitte des Herzens. Während der Vorhof des Schildkrötenherzens, wenn er vom übrigen Herzen abgetrennt wird, sich in der alten Periode weiter kontrahirt, tritt hei dem abgetrennten Ventrikel ein Stillstand ein. Das beweist aber nicht, dass der Ventrikel keiner automatischen Thätigkeit fähig ist, sondern nur, dass die physikalisch-chemischen Bedingungen für die automatische Thätigkeit des Ventrikels etwas andere sind als für den Vorhof. Durch eine Reihe von Arbeiten ist in meinem Laboratoriun der Nachweis erbracht worden, dass die Erregbarkeit von Nerven und Muskeln und die rhythmische Thätigkeit verschiedener Organe unter Anderem eine Function des Quotienten $\frac{\mathrm{C}_{\mathrm{sa}}}{\mathrm{C}_{\mathrm{Ca}}}$, d. h. der Concentration der Natriumionen dividirt durch die Concentration der Calciumionen der umgebenden Lösung, resp. der betreffenden Gewebe ist. Im Blute der Schildkröte ist der Wert dieses Quotienten gross genug, um die rhythmischen Contractionen des Vorhofs zu erlauben, aber zu klein, um die rhythmischen Contractionen des Ventrikels zu gestatten ${ }^{2}$. Aehnlich liegen die Dinge für die rhythmischen Contractionen einer Meduse (Gonionemus). Trennt man den Rand vom Centrum dieser Meduse,

1) Die Bezeichnung "noeud vital" sollte aus der Physiologie verschwinden.

2) Der letztere kann also im Körper sich nur dann rhythmisch contrahiren, so lange er mit dem Vorhof zusammenhängt und von diesem seine Impulse erhält. 
540) Jacques Loeb: Ueber den segm. Charakter des Athemcentrums etc.

so ist nur der erstere im Stande, im Seewasser zu schlagen. Erhöht man aber den Werth des Quotienten $\frac{\mathrm{Csa}}{\mathrm{Cca}}$ im Seewasser durch Verringerung der Concentration der Calciumionen oder durch Erhöhung des Werthes der Natriumionen, so treten rhythmische Contractionen des Centrums auf ${ }^{1}$ ).

Ich halte es für möglich, dass etwas Aehnliches für die segmentalen Athemganglien zutrifft. Es mag sein, dass etwas in der Zusammensetzung des Blutes den obersten segmentalen AthemGanglien, den Kiemenganglien, zwar erlaubt, ihre automatische Thätigkeit auszuführen, während das für die cervicalen und thoracalen Athemganglien nicht der Fall ist ${ }^{2}$ ).

1) Diese Thatsachen sind durch eine Reihe von Versuchen in meinem Laboratorium festgestellt und in den letzten Bänden des Amer. Journal of Physiology veröffentlicht worden.

2) Ich habe bereits in der englischen Ausgabe meiner vergleichenden Gehirnphysiologie darauf hingewiesen, dass die physikalisch-chemischen Bedingungen für die Thätjgkeit der Kopf- und Rumpfsegmente im Allgemeinen etwas verschieden sind, wie das ja auch für das Centrum und den Rand von Gonionemus der Fall ist. 